\title{
Optical Frequency Up-Conversion of the Ferromagnetic Resonance in an Ultrathin Garnet Mediated by Magnetoelastic Coupling
}

\author{
Lucile Soumah $\odot,{ }^{1}$ Davide Bossini $\odot,{ }^{2}$ Abdelmadjid Anane, ${ }^{3}$ and Stefano Bonetti $\odot^{1,4, *}$ \\ ${ }^{1}$ Department of Physics, Stockholm University, 10691 Stockholm, Sweden \\ ${ }^{2}$ Department of Physics and Center for Applied Photonics, University of Konstanz, 78464 Konstanz, Germany \\ ${ }^{3}$ Unit Mixte de Physique CNRS, Thales, Université Paris-Sud, Université Paris Saclay, 91767 Palaiseau, France \\ ${ }^{4}$ Department of Molecular Sciences and Nanosystems, Ca' Foscari University of Venice, 30172 Venice, Italy
}

(Received 30 November 2020; revised 7 June 2021; accepted 19 July 2021; published 11 August 2021)

\begin{abstract}
We perform ultrafast pump-probe measurements on a nanometer-thick crystalline Bi-doped yttrium iron garnet film with perpendicular magnetic anisotropy. Tuning the photon energy of the pump laser pulses above and below the material's band gap, we trigger ultrafast optical and spin dynamics via both one- and two-photon absorption. Contrary to the common scenario, the optically induced excitation induces an increase up to $20 \%$ of the ferromagnetic resonance frequency of the material. We explain this unexpected result in terms of a modification of the magnetic anisotropy caused by a long-lived photo-induced strain, which transiently and reversibly modifies the magnetoelastic coupling in the material. Our results disclose the possibility to optically increase the magnetic eigenfrequency in nanometer-thick magnets.
\end{abstract}

DOI: 10.1103/PhysRevLett.127.077203

Since the discovery of the giant magnetoresistance effect [1], static equilibrium magnetization states are the most common method to encode digital information in data centers worldwide. To date, the dynamical properties of magnetization remain mostly unexploited in commercial technologies, with the notable exception of microwave frequency devices such as garnet-based oscillators [2]. Magnetization dynamics is attractive for technology since the frequency of magnetization precession can be tuned from the gigahertz to the terahertz range by the choice of different materials or by tuning an externally applied magnetic field [3]. Collective spin excitations-so-called spin waves-can also be used to write, transport, or manipulate magnetic information $[4,5]$. The best media to excite and propagate spin waves are materials with low Gilbert damping $\alpha$, with bulk yttrium iron garnet (YIG) holding the record for the lowest value $\alpha=5 \times 10^{-5}[6,7]$, hence a preferred choice for spin wave devices [8]. Nowadays it is even possible to grow nanometer-thick, high-quality crystalline garnet films with comparable Gilbert damping of $\alpha=6 \times 10^{-5}$ [9-12], outperforming that of metallic magnetic films by more than an order of magnitude [13]. These ultrathin garnet films are key to study both fundamental and applied problems of spin wave dynamics at the nanoscale [7,14-16].

Published by the American Physical Society under the terms of the Creative Commons Attribution 4.0 International license. Further distribution of this work must maintain attribution to the author(s) and the published article's title, journal citation, and DOI. Funded by Bibsam.
Ultrashort light pulses have emerged as an efficient tool to initiate and probe magnetization dynamics in iron garnets on the (sub)picosecond timescale [17-20]. The numerous studies on optically induced magnetization dynamics in garnets have unraveled both dissipative $[21,22]$ and nondissipative $[23,24]$ mechanisms to trigger magnetization precession, magnetization reversal [25], and spin wave propagation [26]. Dissipative processes rely on the absorption of light via allowed electronic transitions, while nondissipative mechanisms can be realized when photon energies tuned far away from these transitions are used. In the latter case, light-scattering mechanisms such as impulsive stimulated Raman scattering couple the electric field of light to the spin system without heat transfer [27]. This large research effort has hitherto been mostly focused on relatively thick iron garnets $[20,26,28]$. On the other hand, ultrathin magnetic garnets have been barely studied as the ultrafast spin dynamics is concerned [24]. Despite the potential of scaling the generation, manipulation, and detection of coherent magnetization precession to the nanoscale $[15,16,29]$, the ultrafast spin dynamics in fewnanometer-thick garnet films with perpendicular magnetic anisotropy (PMA) and low magnetic losses has not been investigated yet. We note that it follows from the thermodynamical description of a magnetic material and spin wave theory, that laser-induced heating can decrease the magnetic eigenfrequency, as the sample temperature increases. However, increasing the frequency of collective spin dynamics in a given material without relying on a tunable external field is crucial for technological applications, since it implies both a larger bandwidth operation as well as a lower $1 / f$ noise at the given operating frequency. 
In this Letter, we study light-induced magnetization dynamics in a nanometer-thick Bi:YIG film with PMA. We use two different pump wavelengths to trigger magnetization dynamics with photon energies above and below the material band gap [30,31]. To access both optical absorption and light-induced spin dynamics, we measure the transient transmissivity as well as the rotation of the probe polarization. The time-resolved transmissivity data show that the sample is laser heated, via different mechanisms depending on the pump wavelength. The rotation of the polarization, due to the magneto-optical Faraday effect, reveals that the ferromagnetic resonance is triggered by laser-induced heating. Remarkably, we succeed in increasing the frequency of the magnetic precession up to $20 \%$ of its initial value by tuning the fluence of the photoexcitation.

The Bi:YIG sample studied in this Letter is $17 \mathrm{~nm}$ thick and was grown by pulsed laser deposition on a (111) oriented, substituted gadolinium gallium garnet substrate. The PMA in this sample is stabilized by both magnetoelastic and growth-induced anisotropy [10]. Bismuth-doping enhances the magneto-optical response compared to pristine YIG [32]. The experimental setup is described in the Supplemental Material [33]. In order to investigate the optical excitation with photon wavelength tuned below and above the band gap, we used two different wavelengths: 800 and $400 \mathrm{~nm}$, whose corresponding photon energies $(1.55$ and $3.1 \mathrm{eV})$ are well below and slightly above the Bi:YIG band gap at approximately $3 \mathrm{eV}$ [30,31]. All signals are normalized with respect to the measured optical intensity. The rotation of the probe polarization is additionally normalized in respect to the magneto-optical hysteresis loops, detected in the same setup. Such normalization is necessary to convert the rotation of polarization in units of transient changes of the saturation magnetization [33]. We label these normalizing signals $T_{0}$ for transmissivity and $\theta_{0}$ for the rotation. Minimal cross talking between pump and probe beams is achieved, as they always have different wavelengths, either 800 or $400 \mathrm{~nm}$. In iron garnets, the magneto-optical response of each iron sublattice varies within the optical range. The absorption of the material is greatly increased above the band gap, and the literature reports values that differ by at least one order of magnitude for 400 and $800 \mathrm{~nm}$ [26,32]. We could estimate the absorbance of our films to be approximately $5 \pm 1 \%$ at $400 \mathrm{~nm}$. At $800 \mathrm{~nm}$ the absorbance value was below our experimental sensitivity of approximately $1 \%$ for these films, consistent with the literature. Therefore, in order to perform experiments at comparable absorbed fluences in correspondence of the two pump wavelengths, we set the incident fluence of the $800 \mathrm{~nm}$ pump beam to a value 10 times higher than the $400 \mathrm{~nm}$ one.

The temporal traces of the pump-induced changes in transmissivity $\left(\Delta T / T_{0}\right)$ for 800 and $400 \mathrm{~nm}$ pump wavelengths are displayed in Figs. 1(a) and 1(b). For the $800 \mathrm{~nm}$ pump, $\Delta T / T_{0}$ increases sharply at the temporal overlap of
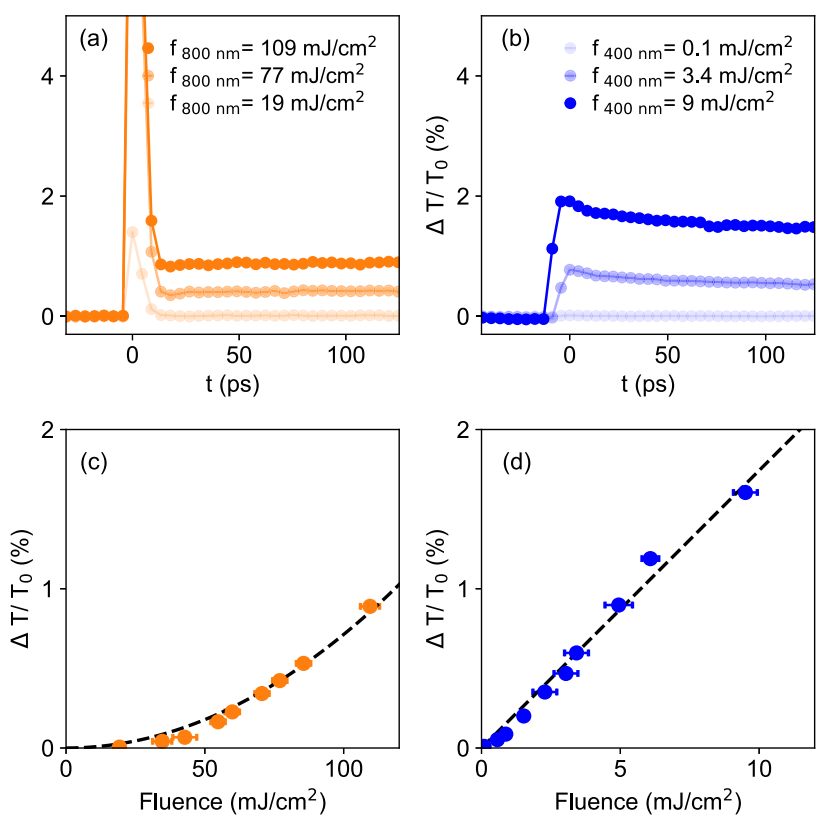

FIG. 1. Temporal evolution of the transient transmissivity of the Bi:YIG film for (a) 800 and (b) $400 \mathrm{~nm}$ pump excitation. Fluence dependence transmissivity 100 ps after the temporal overlap for (c) 800 and (d) $400 \mathrm{~nm}$ pump excitation wavelengths. Dashed lines are fits to the curves, described in detail in the main text.

pump and probe pulses, then it quickly relaxes down to the negative delays value. A substantial increase from the unpumped baseline is clearly observed for fluences equal and higher than $50 \mathrm{~mJ} / \mathrm{cm}^{2}$ [Figs. 1(a) and 1(c)]. For the $400 \mathrm{~nm}$ pump data shown in Fig. 1(b), the transmissivity does not show the same sharp increase at time zero, but it relaxes on similar timescales of hundreds of picoseconds.

In order to get more insight on the pump absorption processes, we evaluate the change in transmissivity $100 \mathrm{ps}$ after the overlap as a function of the fluence. This is shown in Figs. 1(c) and 1(d) for 800 and $400 \mathrm{~nm}$, respectively. For the $800 \mathrm{~nm}$ pump, $1.55 \mathrm{eV}$, the fluence dependence of $\Delta T / T_{0}$ can be well fitted by a quadratic function. Such dependence is consistent with a two-photon absorption process, commonly reported for garnets optically excited at this wavelength $[17,21,23,26]$. Conversely, $\Delta T / T_{0}$ for the $400 \mathrm{~nm}$ pump shows a linear fluence dependence, signature of a one-photon absorption process. For a photon energy of approximately $3.1 \mathrm{eV}$, the absorption of one photon involves a direct electronic transition from the ground to the excited state, i.e., the charge transfer transition, which defines the band gap [26,30,31]. The long-lasting increase of the transmissivity after time zero suggests that, at both pump wavelengths, the laser pulses may induce a temperature increase in the sample. We point out that the same electronic transition is involved in both the 400 and $800 \mathrm{~nm}$ absorption processes. Indeed, no crystal field transitions are excited by $1.55 \mathrm{eV}$ photons [32], but only the charge transfer [30]. 

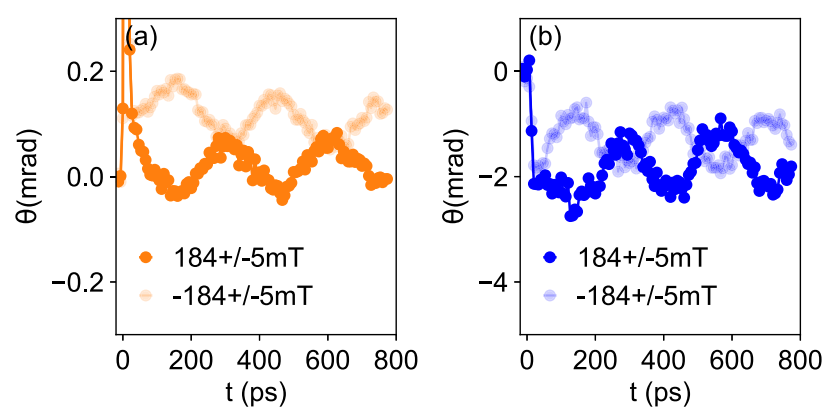

FIG. 2. Time-resolved Faraday rotation following the excitation using pump pulses (a) 800 and (b) $400 \mathrm{~nm}$ wavelengths, with a fluence of approximately $9 \mathrm{~mJ} / \mathrm{cm}^{2}$ and, respectively, $0.3 \mathrm{~mJ} / \mathrm{cm}^{2}$. The magnitude of the externally applied magnetic field $\mu_{0} H_{\text {ext }}=184 \mathrm{mT}$ was nominally the same, with its direction reverted in the semitransparent symbols.

We now turn the discussion to the time-resolved Faraday rotation (FR) of the polarization. The full response of the sample is shown in Fig. 2, in which the data are plotted in absolute values (milliradians) in order to give a flavor of the magnitude of the signal. For both 800 and $400 \mathrm{~nm}$ pump wavelengths, the FR shows two distinct features: (i) a fast $(<5 \mathrm{ps})$ offset that is mostly independent of the direction of the applied magnetic field, and (ii) well-defined and long-lived ( $>100 \mathrm{ps}$ ) oscillations whose phase is reversed upon reversal of the externally applied magnetic field $\mu_{0} H_{\text {ext }}$. This straightforward dependence on the sign of the magnetic field allows one to readily disentangle the nonmagnetic from the magnetic responses, by simply taking the difference between data measured with the magnetic field of opposite polarities.

To understand how the pump absorption triggers this magnetic precession, we study the effect of the pump fluence on the FR signal. The difference between data at opposite fields is plotted in Figs. 3(a) and 3(b) for a few selected pump fluences at both excitation wavelengths. The time traces are fitted with the expression $[23,24,35]$

$$
\theta(t) / \theta_{0}=A_{0} e^{-t / \tau_{0}} \cos \left(2 \pi f_{\text {res }} t+\xi\right)-A_{1} e^{-t / \tau_{1}},
$$

where the first term on the right-hand side (weighted by the factor $A_{0}$ ) describes the oscillation of the magnetization at the frequency $f_{\text {res }}$, with an initial phase $\xi$, damped at the characteristic time $\tau_{0}$. The second term (scaled by the amplitude $A_{1}$ ) describes all other magnetic relaxation phenomena, such as demagnetization, occurring with a time constant $\tau_{1}$.

Figures 3(c) and 3(d) show the extracted $A_{0}$ and $A_{1}$ parameters for the two pump wavelengths. For the $800 \mathrm{~nm}$ data shown in Fig. 3(c), both the magnetic oscillation term $A_{0}$ and the relaxation term $A_{1}$ have approximately the same magnitude and increase linearly with fluence. For the $400 \mathrm{~nm}$ data, $A_{0}$ and $A_{1}$ have a comparable magnitude only for fluences up to $0.7 \mathrm{~mJ} / \mathrm{cm}^{2}$, as shown in the inset of
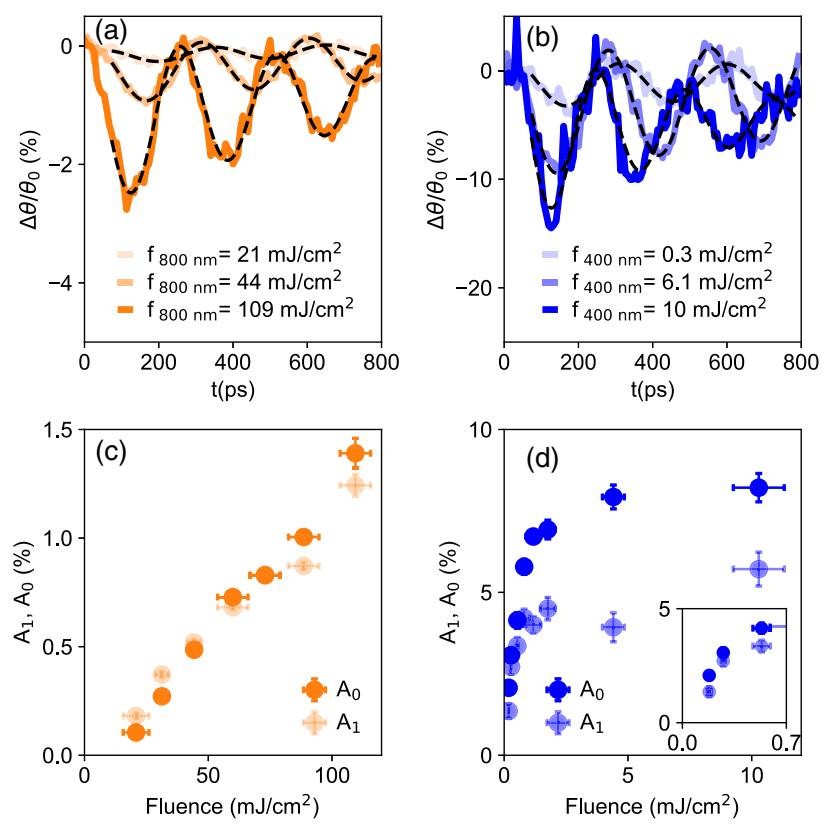

FIG. 3. Time-resolved Faraday rotation data for three different pump fluences at (a) 800 and (b) $400 \mathrm{~nm}$. Dashed lines are fit to the curves using Eq. (1). Fluence dependence of the fitting parameters $A_{0}$ (solid symbols) and $A_{1}$ (semitransparent symbols) in Eq. (1) for (c) 800 and (d) $400 \mathrm{~nm}$ pump wavelengths. Inset: enlarged low-fluence region of (d).

Fig. 3(d). However, for higher fluences, shown in the main panel of Fig. 3(d), the amplitude $A_{0}$ of the magnetic oscillations increases more rapidly than $A_{1}$ and approaches above $5 \mathrm{~mJ} / \mathrm{cm}^{2}$ a saturation value that is approximately twice as large.

The fit of the magnetic response using Eq. (1) also returns the values of $f_{\text {res }}$ in the range of a few gigahertz, which suggest that the observed oscillations are due to the ferromagnetic resonance (FMR). Since we did not observe any effect of the pump polarization, for both pump wavelengths, neither on the amplitude nor on the phase of the oscillations, we rule out nondissipative excitation mechanisms. This would suggest that the FMR precession is heat driven $[17,22,35,36]$. Heat-induced FMR precession in magnetic garnets often is attributed to ultrafast heating of the lattice, which causes a sudden change in the magnetic anisotropy [22]. In turn, this modification of the anisotropy results in a misalignment between the effective magnetic field and the magnetization, which is no longer in equilibrium. The recovery dynamics toward the equilibrium state is described by the Landau-Lifshitz-Gilbert equation. However, while the general mechanism of anisotropy modification remains valid, as we discuss below, heat alone cannot explain the magnitude of the effect that we observe, and an additional mechanism must be taken into account.

In order to understand the dynamics in more detail, we now analyze the mechanism inducing the FMR relying on 

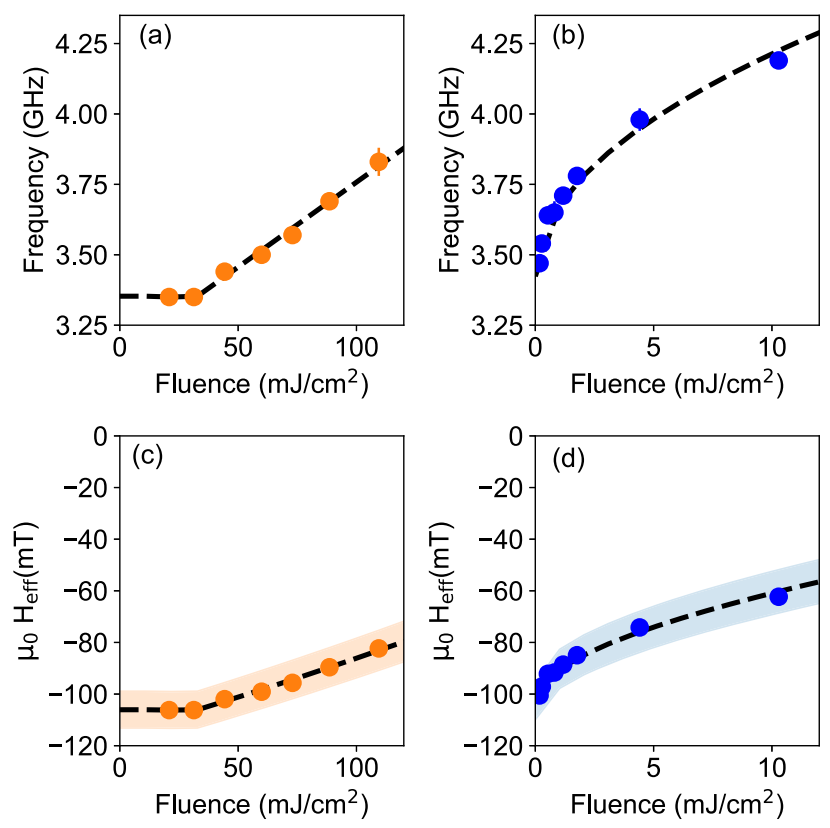

FIG. 4. Extracted FMR frequencies for different pump fluences at (a) 800 and (b) $400 \mathrm{~nm}$ wavelengths. Fluence dependence of the effective field $\mu_{0} H_{\text {eff }}$ calculated using Eq. (2) for (c) 800 and (d) $400 \mathrm{~nm}$ pump wavelengths. Dashed lines in all panels are fit to the curves using the expressions given in the main text. The shaded area indicates the uncertainty in the estimate of the effective field.

the Kittel formalism, which we assume to be valid on the relevant timescales (tens to hundreds of picoseconds). In this temporal regime, a dielectric can be considered to be in an equilibrium state, with an increased temperature in comparison with the state prior to the photoexcitation. From the dependence of the resonant frequency on the external field, we extracted the effective magnetic field of our sample using the Kittel formula $f_{\text {res }}=$ $\gamma \mu_{0} \sqrt{H_{\text {ext }} \cdot\left(H_{\text {ext }}+H_{\text {eff }}\right)}$ [34]. Here, $\gamma \approx 28 \mathrm{GHz} / \mathrm{T}$ s the gyromagnetic ratio, and the effective field is $H_{\text {eff }}=M_{s}-H_{K_{U}}$, with $M_{s}$ being the saturation magnetization and $H_{K_{U}}=2 K_{U} / M_{s}$ the uniaxial anisotropy, with $K_{U}$ being the anisotropy constant. The in plane magnetocrystalline anisotropy in our film is negligible [10]. The estimation of $H_{\text {eff }}$ is described in detail in the Supplemental Material [33], with the fit returning $\mu_{0} H_{\text {eff }} \approx-110 \pm$ $8 \mathrm{mT}$. Such negative value of $\mu_{0} H_{\text {eff }}$ is consistent with a strong PMA, which compensates the dipolar demagnetizing term. Furthermore, from Figs. 3(a) and 3(b), it can be noticed that $f_{\text {res }}$ increases as the pump fluence $F$ increases. The fluence dependence of $f_{\text {res }}$, extracted from Eq. (1), is shown in Figs. 4(a) and 4(b) for 800 and $400 \mathrm{~nm}$ pump wavelengths.

The increase in $f_{\text {res }}$ with fluence is qualitatively different for the two cases. For the $800 \mathrm{~nm}$ pump, the FMR resonance frequency is constant up to $F=50 \mathrm{~mJ} / \mathrm{cm}^{2}$, while for higher fluences it shows a linear increase. At the $400 \mathrm{~nm}$ pump, $f_{\text {res }}$ varies as the square root of $F$ even for the lowest fluence values. The fit of the overall dependence (dashed lines) uses the functions $f_{800}=5 \mathrm{GHz}\left(\mathrm{mJ} / \mathrm{cm}^{2}\right)^{-1} F+$ $3.36 \mathrm{GHz}$ and $f_{400}=0.23 \mathrm{GHz}\left(\mathrm{mJ} / \mathrm{cm}^{2}\right)^{-1 / 2} \sqrt{F}+$ $3.42 \mathrm{GHz}$. The relative error is of the order of $1 \%$ for all extracted parameters. As we kept the direction and the magnitude of the magnetic field constant, the change in resonance frequency must be, according to the Kittel formula, attributed to a photo-induced change in the effective field $\mu_{0} H_{\text {eff }}$ due to the optical pump. Such change of $\mu_{0} H_{\text {eff }}$ is written as

$$
\mu_{0} H_{\text {eff }}=\mu_{0} H_{\text {ext }}-\left(\frac{f_{\text {res }}}{\gamma}\right)^{2} \frac{1}{\mu_{0} H_{\text {ext }}} .
$$

In Figs. 4(c) and 4(d) we plot $\mu_{0} H_{\text {eff }}$ calculated from the data in Figs. 4(a) and 4(b) using Eq. (2). Because of a systematic $5 \mathrm{mT}$ uncertainty on the evaluation of $\mu_{0} H_{\text {ext }}$, $\mu_{0} H_{\text {eff }}$ is estimated with a standard deviation given by the filled region of Figs. 4(c) and 4(d). For both wavelengths, the magnitude of $\mu_{0} H_{\text {eff }}$ decreases with fluence, suggesting that the optical pump effectively quenches the anisotropy of the film. Since the effective field includes both the magnetization and the anisotropy, we cannot exclude a concurring demagnetization of the sample. However, since the frequency is monotonously increasing, the reduction of $M_{s}$ has to be smaller than the reduction $H_{K_{U}}$. Such a decrease is qualitatively consistent with the temperature dependence of the magnetization and the uniaxial magnetic anisotropy constant $\mathrm{K}_{U}$, which is generally expressed as $K_{U}(T) / K_{U}(0 \mathrm{~K})=\left[M_{s}(T) / M_{s}(0 \mathrm{~K})\right]^{3}$ [37].

Given the phenomenological character of these arguments, it is useful to quantify the effect of temperature on the magnetization dynamics. Hence, we perform two additional measurements that we show in the Supplemental Material [33]. At first, we measure the optically induced demagnetization by saturating the magnetization out of plane (i.e., parallel to the laser beam). The maximum demagnetization observed amounts to $2 \%$ and $12 \%$ for the largest fluences used for the $800 \mathrm{~nm}$ and, respectively, the $400 \mathrm{~nm}$ pump beam. The timescale at which the demagnetization was observed is the same as in the case of the data shown in Figs. 2 and 3. Temperature-dependent magnetization curves of similar films [32] allow us to estimate a corresponding temperature increase of few degrees kelvin (800 nm pump, max fluence) and $+50 \mathrm{~K}$ (400 nm pump, max fluence) from room temperature. Then, we estimate $\mu_{0} H_{\text {eff }}$ using microwave-based broadband FMR measurements from 300 to $400 \mathrm{~K}$, covering and exceeding the temperature range reached by the thin film in the pump-probe experiment. The FMR data show a change in effective field of approximately $+20 \mathrm{mT}$ at $400 \mathrm{~K}$ and of $+10 \mathrm{mT}$ at $350 \mathrm{~K}$. Remarkably, this is much smaller than the optically induced modification of the effective field shown in Fig. 4. In particular, it shows that temperature can 
only account for approximately a fifth of the observed variation in $\mu_{0} H_{\text {eff }}$ for the $400 \mathrm{~nm}$ pump at the largest fluence, and it is a negligible contribution in the case of $800 \mathrm{~nm}$ pump experiments.

Recently, a time-resolved diffraction experiment using optical pump and X-ray pulses as probe reported a longlived (ns) strain on a similar Bi:YIG film as the one investigated in our work, but much thicker and without PMA [38]. A similar quadratic and linear dependence of the strain on the fluence for $800 \mathrm{~nm}$ and, respectively, $400 \mathrm{~nm}$ pump pulses was observed. There are, therefore, no reasons to exclude that a nanosecond-long strain is photo-induced in our measurements as well. It is instructive to estimate the possible strain induced from the measured frequency shift and by the known strain of $0.5 \%$ and magnetoelastic field $H_{\mathrm{KMO}} \approx 82 \mathrm{mT}$ for similar films [10]. The largest effective field variation measured in our work is 48 and $28 \mathrm{mT}$ for the $400 \mathrm{~nm}$ and, respectively, $800 \mathrm{~nm}$ pump. Assuming that a variation in the magnetoelastic field would explain all the FMR frequency shifts, this would imply a maximum transient strain of $0.3 \%$ and $0.16 \%$ for the two largest 400 and $800 \mathrm{~nm}$ fluences. These values are comparable to those reported in Ref. [38] for the same pump wavelengths $(0.25 \%$ and $0.08 \%)$ and almost identical fluences. Hence, we identify optically induced strain as the primary cause of the FMR frequency up-conversion that we report.

We briefly comment on the significance of our results. It is remarkable that optical laser pulses are able to quench the effective field of an iron garnet by up to $44 \%$ in a fully reversible way without permanent damage. With sample engineering, it is reasonable to expect that photo-induced full quenching of the magnetic anisotropy may be possible. The all-optical, tunable control of strain has also unique advantages, as standard mechanical means would be practically more complicated to implement, possibly not as reversible, and definitely orders of magnitude slower. We also highlight that the strain-induced frequency up-conversion is quantitatively significant (up to $20 \%$ ), tunable, but also counterintuitive, since noncoherent effects tend to lower the magnetic eigenfrequencies. In our case, due to the significant contribution of the uniaxial anisotropy term in the effective field and to the magnetoelastic coupling, we observe the opposite behavior.

We are not aware of other material systems where a similar phenomenon has been achieved. We observe that, although nonlinear spin dynamics has already been reported in a limited variety of dielectric magnetic materials, the mechanisms are different than the concept of longlived but reversible strain discussed here. In particular, rather different mechanisms such as coupling with lattice oscillations [39] and strong terahertz resonant [40,41] or nonresonant [42] pumping were exploited to induce harmonics of the spin resonances. Hence, our findings reveal a novel mechanism of anisotropy control that can only be achieved in ultrathin iron garnets with PMA. Given that these garnets also possess an extremely low magnetic damping, our results pave the way for new control schemes in spin wave devices where their operating frequencies can be controlled by light.

L. S. and S. B. acknowledge support from the Knut and Alice Wallenberg Foundation, Grant No. 2017.0158. D. B acknowledges support from the Deutsche Forschungsgemeinschaft (DFG) program BO 5074/1-1. We acknowledge support from the COST Action CA17123 "Ultrafast opto-magneto-electronics for nondissipative information technology" (MAGNETOFON).

*stefano.bonetti@fysik.su.se

[1] M. N. Baibich, J. M. Broto, A. Fert, F. Nguyen Van Dau, F. Petroff, P. Etienne, G. Creuzet, A. Friederich, and J. Chazelas, Phys. Rev. Lett. 61, 2472 (1988).

[2] W. S. Ishak and K.-W. Chang, IEEE Trans. Microwave Theory Tech. 34, 1383 (1986).

[3] A. G. Gurevich and G. A. Melkov, Magnetization Oscillations and Waves (CRC Press, Boca Raton, 1996).

[4] A. V. Chumak, A. A. Serga, and B. Hillebrands, Nat. Commun. 5, 4700 (2014).

[5] T. Fischer, M. Kewenig, D. Bozhko, A. Serga, I. Syvorotka, F. Ciubotaru, C. Adelmann, B. Hillebrands, and A. Chumak, Appl. Phys. Lett. 110, 152401 (2017).

[6] H. Yu, O. d. Kelly, V. Cros, R. Bernard, P. Bortolotti, A. Anane, F. Brandl, R. Huber, I. Stasinopoulos, and D. Grundler, Sci. Rep. 4, 6848 (2014).

[7] M. Collet, X. De Milly, O.d. Kelly, V. V. Naletov, R. Bernard, P. Bortolotti, J. B. Youssef, V. Demidov, S. Demokritov, J. Prieto et al., Nat. Commun. 7, 10377 (2016).

[8] A. Serga, A. Chumak, and B. Hillebrands, J. Phys. D 43, 264002 (2010).

[9] C. Hauser, T. Richter, N. Homonnay, C. Eisenschmidt, M. Qaid, H. Deniz, D. Hesse, M. Sawicki, S. G. Ebbinghaus, and G. Schmidt, Sci. Rep. 6, 20827 (2016).

[10] L. Soumah, N. Beaulieu, L. Qassym, C. Carrétéro, E. Jacquet, R. Lebourgeois, J. B. Youssef, P. Bortolotti, V. Cros, and A. Anane, Nat. Commun. 9, 3355 (2018).

[11] T. Yoshimoto, T. Goto, K. Shimada, B. Iwamoto, Y. Nakamura, H. Uchida, C. A. Ross, and M. Inoue, Adv. Electron. Mater. 4, 1800106 (2018).

[12] M. Onbasli, A. Kehlberger, D. H. Kim, G. Jakob, M. Kläui, A. V. Chumak, B. Hillebrands, and C. A. Ross, APL Mater. 2, 106102 (2014).

[13] A. J. Lee, J. T. Brangham, Y. Cheng, S. P. White, W. T. Ruane, B. D. Esser, D. W. McComb, P. C. Hammel, and F. Yang, Nat. Commun. 8, 1 (2017).

[14] M. Collet, O. Gladii, M. Evelt, V. Bessonov, L. Soumah, P. Bortolotti, S. Demokritov, Y. Henry, V. Cros, M. Bailleul et al., Appl. Phys. Lett. 110, 092408 (2017).

[15] H. Merbouche, M. Collet, M. Evelt, V. E. Demidov, J. L. Prieto, M. Muñoz, J. Ben Youssef, G. de Loubens, O. Klein, S. Xavier et al., ACS Appl. Nano Mater 4, 121 (2021).

[16] M. Evelt, L. Soumah, A. Rinkevich, S. Demokritov, A. Anane, V. Cros, J. B. Youssef, G. De Loubens, O. Klein, P. Bortolotti et al., Phys. Rev. Applied 10, 041002(R) (2018). 
[17] F. Hansteen, A. Kimel, A. Kirilyuk, and T. Rasing, Phys. Rev. Lett. 95, 047402 (2005).

[18] A. Kirilyuk, A. V. Kimel, and T. Rasing, Rev. Mod. Phys. 82, 2731 (2010).

[19] I. Yoshimine, T. Satoh, R. Iida, A. Stupakiewicz, A. Maziewski, and T. Shimura, J. Appl. Phys. 116, 043907 (2014).

[20] I. Savochkin, M. Kozhaev, A. Chernov, A. Kuzmichev, A. Zvezdin, and V. Belotelov, Phys. Solid State 59, 904 (2017).

[21] M. Deb, E. Popova, M. Hehn, N. Keller, S. Petit-Watelot, M. Bargheer, S. Mangin, and G. Malinowski, Phys. Rev. Lett. 123, 027202 (2019).

[22] L. A. Shelukhin, V. V. Pavlov, P. A. Usachev, P. Y. Shamray, R. V. Pisarev, and A. M. Kalashnikova, Phys. Rev. B 97, 014422 (2018).

[23] B. Koene, M. Deb, E. Popova, N. Keller, T. Rasing, and A. Kirilyuk, J. Phys. Condens. Matter 28, 276002 (2016).

[24] L. Q. Shen, L. F. Zhou, J. Y. Shi, M. Tang, Z. Zheng, D. Wu, S. M. Zhou, L. Y. Chen, and H. B. Zhao, Phys. Rev. B 97, 224430 (2018).

[25] A. Stupakiewicz, K. Szerenos, M. Davydova, K. Zvezdin, A. Zvezdin, A. Kirilyuk, and A. Kimel, Nat. Commun. 10, 612 (2019).

[26] Y. Hashimoto, D. Bossini, T. H. Johansen, E. Saitoh, A. Kirilyuk, and T. Rasing, Phys. Rev. B 97, 140404(R) (2018).

[27] D. Bossini, A. M. Kalashnikova, R. V. Pisarev, T. Rasing, and A. V. Kimel, Phys. Rev. B 89, 060405(R) (2014).

[28] M. Deb, E. Popova, M. Hehn, N. Keller, S. Petit-Watelot, M. Bargheer, S. Mangin, and G. Malinowski, Phys. Rev. Applied 12, 044006 (2019).

[29] C. Safranski, I. Barsukov, H. K. Lee, T. Schneider, A. Jara, A. Smith, H. Chang, K. Lenz, J. Lindner, Y. Tserkovnyak et al., Nat. Commun. 8, 117 (2017).

[30] S. Wittekoek, T. J. Popma, J. Robertson, and P. Bongers, Phys. Rev. B 12, 2777 (1975).
[31] M. Deb, E. Popova, A. Fouchet, and N. Keller, J. Phys. D 45, 455001 (2012).

[32] P. Hansen and J.-P. Krumme, Thin Solid Films 114, 69 (1984).

[33] See Supplemental Material at http://link.aps.org/ supplemental/10.1103/PhysRevLett.127.077203 for (i) details of the pump-probe setup, (ii) magneto-optical hysteresis loops, (iii) effective field from pump-probe FMR measurements, (iv) optically induced demagnetization, (v) temperature-dependent effective field estimates from broadband microwave FMR measurements, and (vi) estimation of the relative variation of magnetic moment, effective field, and anisotropy, which includes Refs. [31,32,34].

[34] M. Farle, Rep. Prog. Phys. 61, 755 (1998).

[35] M. Deb, M. Vomir, J.-L. Rehspringer, and J.-Y. Bigot, Appl. Phys. Lett. 107, 252404 (2015).

[36] M. Deb, E. Popova, M. Hehn, N. Keller, S. Mangin, and G. Malinowski, Phys. Rev. B 98, 174407 (2018).

[37] C. Zener, Phys. Rev. 96, 1335 (1954).

[38] S. P. Zeuschner, J.-E. Pudell, A. von Reppert, M. Deb, E. Popova, N. Keller, M. Rössle, M. Herzog, and M. Bargheer, Phys. Rev. Research 2, 022013(R) (2020).

[39] D. Afanasiev, I. Razdolski, K. M. Skibinsky, D. Bolotin, S. V. Yagupov, M. B. Strugatsky, A. Kirilyuk, T. Rasing, and A. Kimel, Phys. Rev. Lett. 112, 147403 (2014).

[40] S. Baierl, J. H. Mentink, M. Hohenleutner, L. Braun, T.-M. Do, C. Lange, A. Sell, M. Fiebig, G. Woltersdorf, T. Kampfrath et al., Phys. Rev. Lett. 117, 197201 (2016).

[41] Y. Mukai, H. Hirori, T. Yamamoto, H. Kageyama, and K. Tanaka, New J. Phys. 18, 013045 (2016).

[42] M. Hudl, M. dAquino, M. Pancaldi, S.-H. Yang, M. G. Samant, S. S. P. Parkin, H. A. Dürr, C. Serpico, M. C. Hoffmann, and S. Bonetti, Phys. Rev. Lett. 123, 197204 (2019). 